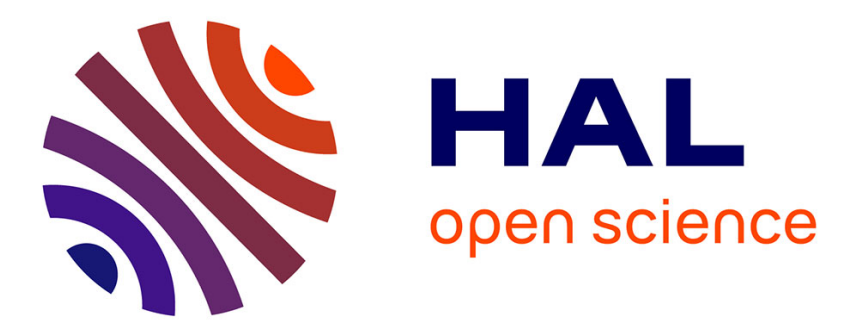

\title{
Ability-Based Optimization: Designing Smartphone Text Entry Interface for Older Adults
}

Jussi Jokinen, Antti Oulasvirta, Xiangshi Ren, Chaklam Silpasuwanchai, Zhenxin Wang, Sayan Sarcar

\section{- To cite this version:}

Jussi Jokinen, Antti Oulasvirta, Xiangshi Ren, Chaklam Silpasuwanchai, Zhenxin Wang, et al.. Ability-Based Optimization: Designing Smartphone Text Entry Interface for Older Adults. 16th IFIP Conference on Human-Computer Interaction (INTERACT), Sep 2017, Bombay, India. pp.326-331, 10.1007/978-3-319-68059-0_22 . hal-01679821

\section{HAL Id: hal-01679821 \\ https://hal.inria.fr/hal-01679821}

Submitted on 10 Jan 2018

HAL is a multi-disciplinary open access archive for the deposit and dissemination of scientific research documents, whether they are published or not. The documents may come from teaching and research institutions in France or abroad, or from public or private research centers.
L'archive ouverte pluridisciplinaire HAL, est destinée au dépôt et à la diffusion de documents scientifiques de niveau recherche, publiés ou non, émanant des établissements d'enseignement et de recherche français ou étrangers, des laboratoires publics ou privés. 


\title{
Ability-based Optimization: Designing Smartphone Text Entry Interface for Older Adults
}

\author{
Sayan Sarcar ${ }^{1}$, Jussi Jokinen ${ }^{2}$, Antti Oulasvirta ${ }^{2}$, Xiangshi Ren ${ }^{1}$, Chaklam \\ Silpasuwanchai ${ }^{1}$, Zhenxin Wang $^{1}$ \\ ${ }^{1}$ Center for Human-Engaged Computing, Kochi University of Technology Japan \\ \{sayan.sarcar, ren.xiangshi, chaklam, wang.zhenxin\}@kochi-tech.ac.jp \\ ${ }^{2}$ Aalto University, Finland \\ \{jussi.jokinen, antti.oulasvirta\}@aalto.fi
}

\begin{abstract}
Beside decreasing the abilities, individual difference prevails among older adults, as some individuals are completely healthy at the age 90 while some are not at even 60 . In context of touchscreen interface design, it is critical to understand the design space as a function of abilities. In this work, we articulate a better understanding of the effects of ageing and examine their HCI task performing capabilities in terms of interfaces design. We design a text-entry interface in particular, as ageing users often achieve slow text entry performance, thus proving to be a bottleneck for technology use. Our developed text entry interface is tuned based on the parameter values for Elderly having finger tremor. We present initial study results showing the improvement of the accuracy of touch typing in smartphone over the baseline Qwerty keyboard. By carefully considering other sensorimotor abilities, we believe that the current smartphone text-entry interface designs will become more usable to the ageing populations.
\end{abstract}

Keywords: Ability-based design, aging users, text entry interface, tremor

\section{Introduction}

By the year 2050, ageing populations are expected to cover $27 \%$ and $15 \%$ of developed and developing nations, respectively [1]. This paper is motivated by the need to design user interfaces (UIs) that better capture age-related changes in motor (dexterity such as tremor, Parkinson's) [8], perceptual (e.g., visual acuity, oculomotor performance)[3], and cognitive abilities (e.g., task-switching). The most striking factor about age-related change is its variability.

When it comes to interface design, the "one design fits all" approach does not work well. For example, the smartphones (DORO ${ }^{T M}$, Fujitsu, Softbank), designed specifically for older adults, got failed to gain popularity within the target community. Are those provided best suitable user interfaces for somebody with, say, issues with tremor but with perfect vision? Thus, there is a clear need to complement interface design with a better understanding of effects of ageing. Although recent works have explored Ability-Based Design concept $[2,4]$ to develop interfaces personalized to the abilities, little has been done to connect the psychological phenomena of ageing to design.

This paper aims to articulate a better understanding of the effects of ageing and examine their HCI significance in interface design. As a case study, we look at text-entry interfaces, given that ageing users were able to only achieve 7-8 WPM text typing speed with onscreen Qwerty keyboard [7] and thus prove to be real bottleneck when using technologies. The major takeaways of this paper: (1) 


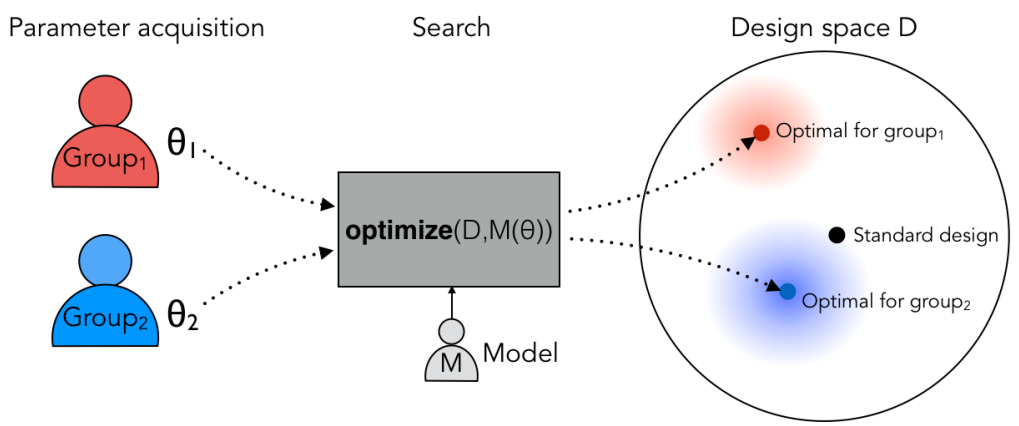

Fig. 1: Overview of parametric optimization of UI in ability-based design

development of a design space for text-entry interfaces and (2) design and initial evaluation of the keyboard interface suitable for older adults having tremor. In a broader sense, our work serve as initial attempt to build a psychological foundation that could guide text-entry design and interface design in general.

\section{Approach to design text entry interface}

This paper extends model-based UI optimization to ability-based design [9]. Figure 1 provides an overview of the approach. Application to ability-based design critically builds upon formulation of objective functions in such a way that individual differences can be expressed as part of the objective function. We here explore the idea to express individual differences as parameters $(\theta)$ of a predictive model. We call this the parametric approach.

This work extends ability-based optimization (first implemented the idea were shown by Gajos et al. (e.g., [2]) from the consideration of motor performance difference. However, they were limited to models of motor performance combined with simple heuristics to describe visual impairments. The problem with heuristics (such as "users with poor vision need a larger font") is that they are not able to resolve trade-offs in design.

Another advancement examined here is the use of rational analysis to predict how a user with given characteristic might start using a UI. We assume that interaction with a UI is associated with a space of possible strategies. One wellknown strategy is speed-accuracy trade off in pointing: a person's decision to be faster at the expense of accuracy, or vice versa, be more accurate but at the expense of speed. The identification of relevant strategy spaces is outside the scope of this paper.

Procedurally, parametric model-based interface optimization has the five steps: (1) defining design space and objective function, (2) building a parametrizable predictive model of user behavior, including strategy adaptation, (3) acquiring parameters to describe abilities of a user group, (4) constructing an efficient combinatorial approach to solve the task and (5) testing the robustness of the design to differing assumptions (e.g., change in parameters or task)

Table 1 describes the design parameters, their value ranges, and their related abilities. Figure 2a depicts the design parameters. We selected this design parameters for its potential effects for ageing users and settled their value ranges through rigorous empirical and calibration studies.

In this work, we only focused on designing optimized text entry interface suitable for older people having finger tremor (essential tremor and parkinson's). 
Table 1: Design space and value ranges. ${ }^{*}=$ have been studied in previous work [6], $\mathrm{V}=$ vision, $\mathrm{A}=$ Attention, $\mathrm{M} 1=$ Motor, M2=Memory)

\begin{tabular}{|c|c|c|c|}
\hline Area of Interest & Parameter & Range / Possible Values & Related abilities \\
\hline \multirow[t]{6}{*}{ Keyboard } & K1: Key size & {$[26.5 \mathrm{dp}, 177.5 \mathrm{dp}]$} & $\mathrm{V}, \mathrm{M} 1$ \\
\hline & K2: No. of keys in each row & $2,3,4,5,7,9,10$ & $\mathrm{~V}$ \\
\hline & K3: Number of rows & {$[1,3]$} & $\mathrm{V}$ \\
\hline & K4: Space between keys & {$[0 \mathrm{dp}, 10 \mathrm{dp}]$} & $\mathrm{V}, \mathrm{M} 1$ \\
\hline & K5: Grid type & QWERTY, $2 \times 2,3 \times 3,5 \times 2,10 \times 1$ & $\mathrm{~V}, \mathrm{M} 1, \mathrm{M} 2$ \\
\hline & K6: Visual feedback-keypress & 0,1 & $\mathrm{~V}, \mathrm{M} 1, \mathrm{~A}$ \\
\hline \multirow[t]{3}{*}{ Word Prediction } & W1: Key size & $72.5 \mathrm{dp}, 96.6 \mathrm{dp}, 145 \mathrm{dp}$ & $\mathrm{V}, \mathrm{M} 1, \mathrm{~A}$ \\
\hline & W2: No. of keys in each row* & {$[2,4]$} & $\mathrm{V}$ \\
\hline & W3: Number of rows* & {$[1,5]$} & $\mathrm{V}$ \\
\hline \multirow[t]{3}{*}{ Text Display } & D1: Text font size & $25 \mathrm{sp}$ & $\mathrm{V}, \mathrm{A}$ \\
\hline & D2: Deleting strategies & no, letter, word & $\mathrm{V}, \mathrm{M} 1, \mathrm{~A}$ \\
\hline & D3: Number of rows* & {$[2,7]$} & $\mathrm{V}$ \\
\hline
\end{tabular}

Thus, we chose the keyboard UI from design space, through model-based optimization (details are in [6]), to support the users having tremor. The grouped keyboard layout $(3 \times 3$ button grid) with multiple letter arranged into each button has been selected by optimizer following the situation that bigger sized keys are easier to be tapped by users having finger tremor (see Fig. 2b). We considered four design parameters for observing to the model: number of rows in the word prediction list (WPL), row height, number of words to be provided in each row of the list, and number of rows in text display area. It is to be noted that some parameters, which can be derived from the parameters list (e.g., area occupied with the keyboard can be defined, for a specified smartphone size, from key size, space between keys and number of columns in the keyboard; area occupied by the text display from keyboard size, WPL key size and number of rows in WPL list; number of characters to map at one key can be calculated by the grid type, whether $3 \times 3$ or $5 \times 2$ ).

\section{Experiment and initial results}

To judge the efficacy of the grouped layout, we conducted text typing experiment (comparison study) with two older adults (a 69 year aged male (P1) and a 67 year aged female (P2)). Participants performed a calibration study on the Samsung Galaxy S6 smartphone where they were asked to tap as quickly and accurately as possible. Next, we conducted text transcription tasks with two keyboards: baseline Qwerty (Fig. 2a) and grouped keyboard (Fig. 2b). Keyboards were selected in random order and were counterbalanced across participants. In the transcription task, participants were instructed to type a sentence repeatedly for 10 minutes for each keyboard. This repetition ensures the familiarity with both the layouts as our target was to analyze the natural text typing speed and accuracy, not the learnability. Also, we did not envision typing both upper and lower case letters with two layouts, as are interested in investigating atomic level typing speed and accuracy of users. We scheduled a 5-minute breaks after calibration and first transcription task.

First results showed that the average typing speed (in WPM, calculated as [5] was slightly higher for the Qwerty (7.35 WPM) compared to the grouped layout (5.28 WPM). In contrast, the average total error rate was much higher Qwerty (14.20\%) than the grouped keyboard (6.71\%). The reason of error occurrence in the Qwerty layout is improper aiming of tapping the button. The grouped layout supports less typing errors (mainly with inaccurate touch down activity), which suits the behavior of the older adults having essential tremors. 


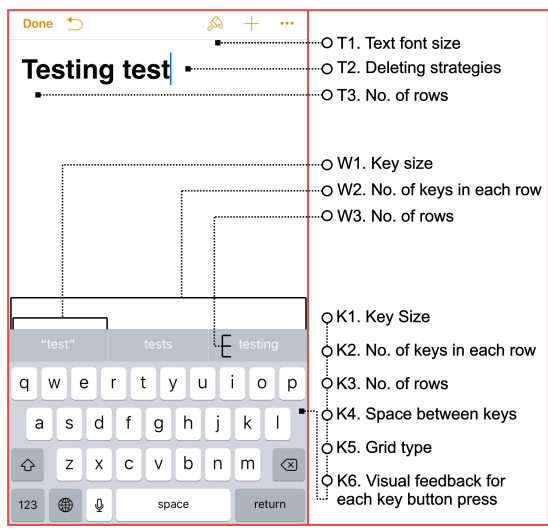

(a)

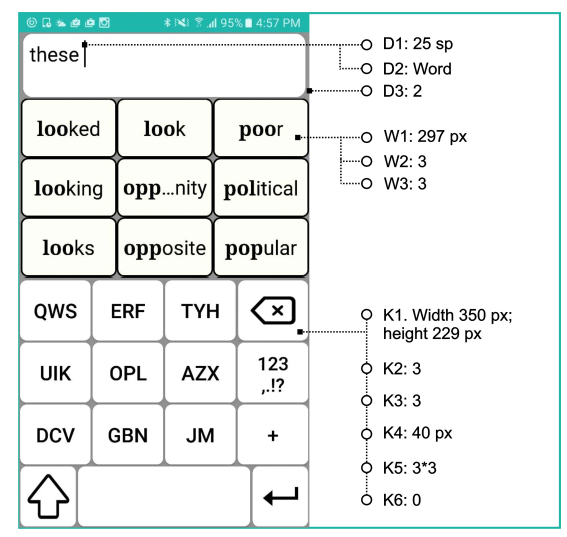

(b)

Fig. 2: Design parameters in (a) Qwerty and (b) Grouped layout design

\section{Conclusion}

This work is based on Ability-Based Optimization concept [6], which refers to the use of computational models to better address individual differences brought about by ageing. By formulating individual differences parametrically and also take into account how users adapt their behavioral strategies as a function of their abilities in a model, this method allows practitioners to investigate optimal designs and to examine trade-offs among several design factors. We believe that combining a scientifically-grounded design space with modeling and optimization can help bridge the gap. One possible next step is to design text entry interfaces considering other sensorimotor abilities and their combinations for older adults.

\section{References}

1. Fisk, A.D., Rogers, W.A., Charness, N., Czaja, S.J., Sharit, J.: Designing for older adults: Principles and creative human factors approaches. CRC press (2009)

2. Gajos, K.Z., Wobbrock, J.O., Weld, D.S.: Improving the performance of motorimpaired users with automatically-generated, ability-based interfaces. In: Proceedings of the SIGCHI conference on Human Factors in Computing Systems. pp. 12571266. ACM (2008)

3. Haegerstrom-Portnoy, G., Schneck, M.E., Brabyn, J.A.: Seeing into old age: vision function beyond acuity. Optometry and vision science : official publication of the American Academy of Optometry 76(3), 141-158 (1999)

4. Hurst, A., Hudson, S.E., Mankoff, J., Trewin, S.: Automatically detecting pointing performance. In: Proceedings of the 13th international conference on Intelligent user interfaces. pp. 11-19. ACM (2008)

5. Nicolau, H., Jorge, J.: Elderly text-entry performance on touchscreens. In: Proceedings of the 14th international ACM SIGACCESS conference on Computers and accessibility. pp. 127-134. ACM (2012)

6. Sarcar, S., Joklnen, J., Oulasvirta, A., Silpasuwanchai, C., Wang, Z., Ren, X.: Towards ability-based optimization for aging users. In: Proc. IxAP 2016. pp. 77-86. ACM (2016)

7. Smith, A.L., Chaparro, B.S.: Smartphone text input method performance, usability, and preference with younger and older adults. Human Factors 57(6), 1015-1028 (2015)

8. Sturman, M.M., Vaillancourt, D.E., Corcos, D.M.: Effects of aging on the regularity of physiological tremor. Journal of neurophysiology 93(6), 3064-3074 (2005)

9. Wobbrock, J.O., Kane, S.K., Gajos, K.Z., Harada, S., Froehlich, J.: Ability-based design: Concept, principles and examples. ACM Transactions on Accessible Computing (TACCESS) 3(3), 9 (2011) 